\title{
Cylindrical Refractive Error
}

National Cancer Institute

\section{Source}

National Cancer Institute. Cylindrical Refractive Error. NCI Thesaurus. Code C117892.

A measurement of the extent of defect in vision caused by a mismatch of optical power between the cylindrical and horizontal meridians, resulting in sub-optimal reflection of light onto the retina. 